\title{
Nonlinear optical transformation of the polarization state of circularly polarized light with holographic-cut cubic crystals
}

\author{
N. Minkovski, ${ }^{1,2}$ S. Kourtev, ${ }^{1, *}$ L. Canova, ${ }^{3}$ A. Jullien, ${ }^{3}$ O. Albert, ${ }^{3}$ and R. Lopez-Martens ${ }^{3}$ \\ ${ }^{1}$ Faculty of Physics, Sofia University, 5 J. Bourchier Boulevard, Sofia-1164, Bulgaria \\ ${ }^{2}$ Department of Physics, University of Forestry, $10 \mathrm{~K}$. Ohridski Boulevard, Sofia-1756, Bulgaria \\ ${ }^{3}$ Laboratoire d'Optique Appliquée, UMR 7639 CNRS, Ecole Polytechnique, ENSTA_Paristech, 91761 Palaiseau Cedex, France \\ ${ }^{*}$ Corresponding author: skourtev@phys.uni-sofia.bg
}

Received October 18, 2010; accepted November 19, 2010;

posted December 14, 2010 (Doc. ID 136742); published January 14, 2011

Nonlinear modification of circularly polarized light propagating in holographic-cut cubic crystals is theoretically predicted and experimentally observed. To the best of our knowledge this is the first demonstration of nonlinear modification of circularly polarized light with cubic crystals. (c) 2011 Optical Society of America

OCIS codes: $190.7110,190.4380,320.7130$.

It is well known that circularly polarized (CP) light cannot change its polarization state nonlinearly when propagating through any isotropic nonlinear media no matter how big the nonlinearity and the intensity are. The same is valid for $z$-cut cubic crystals [1]]. Propagation of intense laser beams through materials and the nonlinear optical transformation of the pulse parameters, including the polarization state, are issues of great importance for many applications. Although in some cases nonlinear change of the pulse properties is a drawback, there are applications where such change is essential. In all cases the knowledge of the light-matter interaction at high intensities is valuable.

At sufficiently high light intensities it is possible to reach the threshold levels of various nonlinear effects, e.g., critical self-focusing and beam filamentation. For most of these effects the polarization state of the incident light is crucial. Generally, the threshold is higher when the light is circularly polarized. Some researchers have systematically investigated the influence of the input polarization state on some of these effects, e.g., multiple filamentation [2], multiphoton ionization and multiphoton excitation [3] , and white-light generation [4]].

It is also well known that for linearly isotropic media the nonlinear change in the index of refraction is minimal for CP incident light compared with any other polarization state (see e.g. [5]) and the critical power for selftrapping of the beam is maximal for CP light. Because optical bulk damage in transparent materials by ultrashort optical pulses is primarily connected with selffocusing, optical damage threshold is higher for CP light.

Circular polarization could be advantageous for pulse compression techniques in gases. Through use of circular polarization, the incident energy in a gas cell can be increased by more than 1.5 times due to the reduction in both the Kerr nonlinearity and the ionization rate of the gas at equivalent intensities. This makes it possible to obtain shorter compressed pulses through filamentation in gases or using gas-filled hollow fibers [6,7].

As we see, circular polarization is an important polarization state for many applications. Therefore the knowl- edge of possible preservation or modification of this polarization state is of great importance.

In recent years we investigated the nonlinear process of cross-polarized wave (XPW) generation. XPW generation became a key effect for temporal and spatial contrast improvement devices in the field of femtosecond laser science and technology. Systematic investigations of this process have been conducted mainly using cubic crystals with linearly and elliptically polarized input beams [8]. In most cases the crystals are oriented along their optical axis ( $z$ cut). Circular polarization is an eigen polarization for $z$-cut cubic crystals and does not change either linearly or nonlinearly. Recently we proposed a different orientation of the cubic crystals, holographic-cut ([110] or equivalent cut) orientation, which gives many advantages over $z$ cut $[\underline{9}, 10]$. The properties of holographic-cut orientation have been investigated only for linearly polarized input. We, however, derived theoretically that the circular polarization state can be modified efficiently with holographic-cut cubic crystals.

Our analysis is based on the full system of differential equations published in [9] that describe the process of XPW generation in plane-wave approximation:

$$
\begin{aligned}
-i \frac{d A(\zeta)}{d \zeta}= & \gamma_{1} A A A^{*}+\gamma_{2} A A B^{*}+2 \gamma_{2} A B A^{*}+2 \gamma_{3} A B B^{*} \\
& +\gamma_{3} B B A^{*}+\gamma_{4} B B B^{*}, \\
-i \frac{d B(\zeta)}{d \zeta}= & \gamma_{5} B B B^{*}+\gamma_{4} B B A^{*}+2 \gamma_{4} A B B^{*}+2 \gamma_{3} A B A^{*} \\
& +\gamma_{3} A A B^{*}+\gamma_{2} A A A^{*} .
\end{aligned}
$$

In the above equations $A$ denotes the complex amplitude of the fundamental (or pump) wave and $B$ is the complex amplitude of the generated orthogonally polarized wave. $\zeta$ is the longitudinal coordinate in the light propagation direction. Nonlinear coupling coefficients $\gamma_{1}$ and $\gamma_{5}$ are responsible for the self-phase modulation (SPM), while $\gamma_{2}$ and $\gamma_{4}$ govern the XPW conversion process from $A$ to $B$ and from $B$ to $A$ waves through the last terms in Eqs. (1b) and (1a), respectively. $\gamma_{3}$ contributes to the 
Table 1. Nonlinear Coupling Coefficients for z-Cut and Holographic-Cut m3m Cubic Crystal ${ }^{a}$

\begin{tabular}{lll}
\hline & \multicolumn{1}{c}{$z$-Cut } & \multicolumn{1}{c}{ Holographic-Cut } \\
\hline$\gamma_{1}$ & $\gamma_{0}\left[1-(\sigma / 2) \sin ^{2}(2 \beta)\right]$ & $\gamma_{0}[1-(\sigma / 4) \cos (2 \beta)+(3 \sigma / 16) \cos (4 \beta)-7 \sigma / 16]$ \\
$\gamma_{2}$ & $-\gamma_{0}(\sigma / 4) \sin (4 \beta)$ & $\gamma_{0}[(\sigma / 8) \sin (2 \beta)-(3 \sigma / 16) \sin (4 \beta)]$ \\
$\gamma_{3}$ & $\gamma_{0}[1 / 3-(\sigma / 4) \cos (4 \beta)-\sigma / 12]$ & $\gamma_{0}[1 / 3-(3 \sigma / 16) \cos (4 \beta)-7 \sigma / 48]$ \\
$\gamma_{4}$ & $\gamma_{0}(\sigma / 4) \sin (4 \beta)$ & $\gamma_{0}[(\sigma / 8) \sin (2 \beta)+(3 \sigma / 16) \sin (4 \beta)]$ \\
$\gamma_{5}$ & $\gamma_{0}\left[1-(\sigma / 2) \sin ^{2}(2 \beta)\right]$ & $\gamma_{0}[1+(\sigma / 4) \cos (2 \beta)+(3 \sigma / 16) \cos (4 \beta)-7 \sigma / 16]$ \\
\hline
\end{tabular}

${ }^{a} \gamma_{0}=6 \pi \chi_{x x x x}^{(3)} / 8 n \lambda$

cross-phase modulation. Nonlinear coupling coefficients depend on the orientation of the nonlinear medium and on the components of the $\chi^{(3)}$ tensor [9]. Equations (1) apply for linearly nonbirefringent media, and hereafter we restrict our considerations to nonlinear crystals (NLCs) that belong to the $m 3 m$ cubic symmetry group. Assuming that the photon energy is below the half bandgap, the $\chi^{(3)}$ tensor is purely real tensor with only two independent components for $m 3 m$ cubic crystals [11]: $\chi_{x x x x}^{(3)}$ and $\chi_{x x y y}^{(3)}$. The relation between them is usually defined [11] as $\sigma=\left(\chi_{x x x x}^{(3)}-3 \chi_{x x y y}^{(3)}\right) / \chi_{x x x x x}^{(3)}$ and is referred to as the anisotropy of the $\chi^{(3)}$ tensor. Nonlinear coupling coefficients for $z$-and holographic-cut $m 3 m$ cubic crystals are summarized in Table 1 , where the angle $\beta$ is the angle between the linear input polarization and the $x$ axis of the crystal.

Notice that generally SPM and XPW generation are not the same for the $A$ and $B$ waves, except in the special case of $z$-cut orientation. As a result of these nonequalities, circular polarization will also be nonlinearly modified with holographic-cut crystals.

We solve numerically the system of equations [Eqs. (1)] assuming an optical scheme shown in Fig. 1. When there are no nonlinear effects, the signal transmitted through this optical scheme is zero.

Simulation results for the plane input wave obtained by solving Eqs. (1) with initial conditions $A_{0}=\left|E_{0}\right| / \sqrt{2}$ and $B_{0}= \pm i\left|E_{0}\right| / \sqrt{2}$ (circular polarization, $\alpha= \pm 45^{\circ}$ ) and $A_{0}=E_{0}$ and $B_{0}=0$ (linear polarization, $\alpha=0^{\circ}$ ) are given in Fig. 2, where XPW efficiency is defined as $|B(L)|^{2} /$ $\left|E_{0}\right|^{2}$. The dimensionless parameter $S$ is proportional to the product of nonlinearity $\times$ input intensity $\times$ nonlinear crystal length. In practice $S$ is limited to approximately 5 by self-action effects [12]. In calculations $\sigma=-1.2$ is used, a value that corresponds to the anisotropy of $\mathrm{BaF}_{2}$ [13]. Polarization-state changes are also drawn in Fig. 2. Initially circular polarization transforms into elliptical polarization with orientation of the ellipse axes dependent on the orientation of the crystalline axes. With further increase of $S$ the polarization becomes purely linear. After that the direction of rotation of the electric-field vector is changed and at sufficiently high $S$ circular polarization with other-handedness could be obtained (i.e., left-handed circular polarization can be

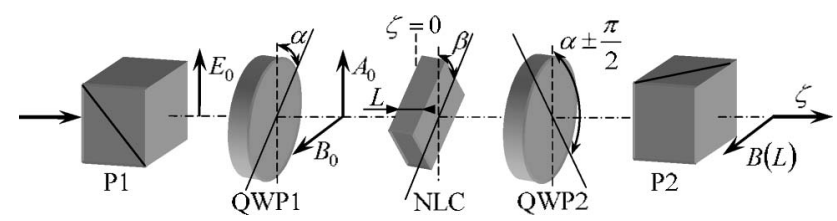

Fig. 1. Optical scheme used. P1, P2, polarizers; QWP, quarter-wave plates; NLC, nonlinear crystal. nonlinearly transformed into right-handed circular polarization and vice versa).

In our experiments we used a Ti:sapphire chirpedpulse amplifier laser system, based on a commercial $1 \mathrm{kHz}$ carrier-envelope phase-stabilized front-end amplifier from Femtolasers $\mathrm{GmbH}$ (Femtopower Compact Pro) followed by a homemade multipass amplifier. To obtain high-fidelity compressed pulses, a feedback loop (DazScope) is applied to an acousto-optic programmable dispersive filter (AOPDF) inside the laser's front end for spectral phase measurement and compensation throughout the entire system [14]. The typical pulse duration after optimization is approximately $25 \mathrm{fs}$.

The experimental setup is as shown in Fig. 1. A $50 \mathrm{~cm}$ focal-length lens placed before the polarizer $(\mathrm{P} 1)$ was used to focus the beam onto the NLC.

Experimental dependences of the output pulse energy on the input pulse energy for both linear and circular input polarizations are shown in Fig. 3. Experimental $\beta$ dependence of the XPW generation efficiency with linearly polarized input pulses is shown in the inset of Fig. 3. The experimental data correspond well to the theoretical curve for holographic-cut crystals. Experimental output pulse energies are not corrected for the linear losses that are caused by reflection from the uncoated surfaces of the crystal and the output Glan. Theoretical curves in Fig. 3 are obtained assuming Gaussian spatial and temporal profiles of the input pulses by numerically solving the system [Eqs. (1)] with initial conditions $E_{0}(r, t) \propto$ $\exp \left(-r^{2}-t^{2}\right)$ followed by numerical integration over $r$

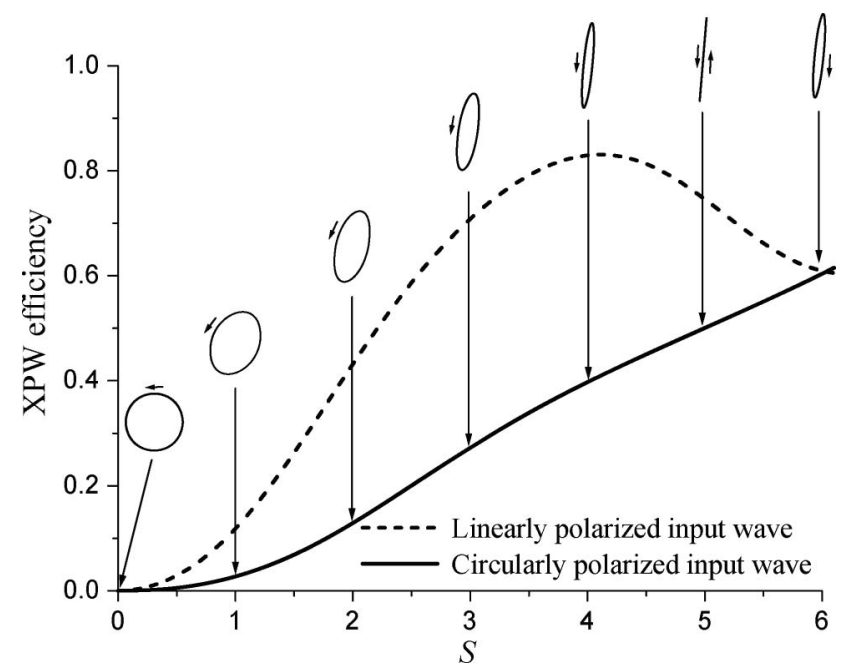

Fig. 2. Simulation results for plane input wave. Solid curve, the efficiency obtained with CP input light. Intensity dependent polarization-state changes after the NLC are also depicted for the corresponding $S$ values. Dashed curve, the efficiency obtained with linear polarization at optimum $\beta=64.5^{\circ}$. 


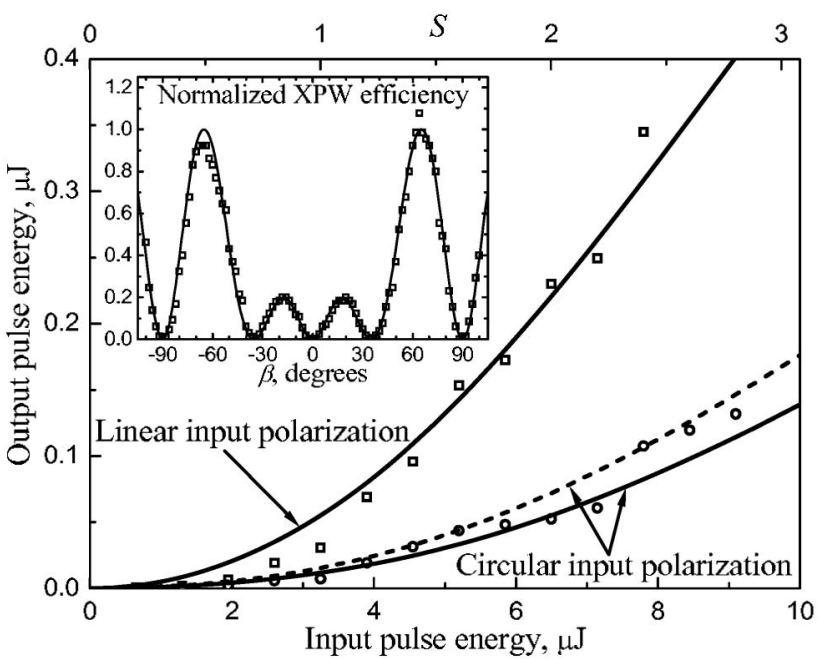

Fig. 3. Experimental energy dependence: squares, with linearly polarized input beam at optimum angle $\beta=64.5^{\circ}$; circles, with CP input beam. Curves are theoretical dependencies. The dashed theoretical curve is obtained for nonperfect circular polarization by using $\lambda / 4 \pm \lambda / 100$ phase plates. $S$ values for the theoretical dependencies are given on the top scale. Inset, $\beta$ scan of the XPW efficiency with linear input polarization; squares, measured with pump energy $6.5 \mu \mathrm{J}$; curve, theoretical simulation with $S=2$.

and $t$. We see a good qualitative agreement between theoretical curves and experimental data. The differences between them are due to propagation effects not included in the model. Such effects might be, for example, change in the beam size due to self-focusing, selfdiffraction, residual and induced chirp, and multiphoton absorption.

It can be seen from Fig. 3 that the XPW generation is more efficient with linearly polarized pump as predicted by the theory. Although the efficiency with circular polarization is less, it is of the same order of magnitude.

The same experiments were conducted with other holographic-cut cubic crystals: $\mathrm{CaF}_{2}$ and $\mathrm{SrF}_{2}$. They showed the same behavior, but rescaled for the different values of the nonlinearity and anisotropy of the crystals.

In conclusion, we show theoretically and prove experimentally that with holographic-cut cubic crystals, CP light can also be nonlinearly modified. As far as we know, it is the first demonstration of nonlinear modification of CP light in cubic crystals.

The research leading to this publication has received funding from the European Commission (EC)'s Seventh Framework Programme (FP7/2007-2013) under grant agreement No. 228334. The authors would also like to acknowledge the support of the Bulgarian National Science Foundation through grant DRNF-02/8/2009 and of the Bulgarian-French collaboration programme RILA-4/209. $\mathrm{N}$. Minkovski and S. Kourtev thank the Laboratoire d'Optique Appliquée for their hospitality and support.

\section{References}

1. D. C. Hutchings and B. S. Werrett, Phys. Rev. B 52, 8150 (1995).

2. G. Fibich and B. Ilan, Phys. Rev. E 67, 036622 (2003).

3. S. Petit, A. Talebpour, A. Proulx, and S. L. Chin, Opt. Commun. 175, 323 (2000).

4. A. K. Dharmadhikari, F. A. Rajgara, and D. Mathur, Appl. Phys. B 80, 61 (2004).

5. R. W. Boyd, Nonlinear Optics, 2nd ed. (Academic, 2003).

6. J. Liu, X. W. Chen, R. X. Li, and T. Kobayashi, Laser Phys. Lett. 5, 45 (2008).

7. X. Chen, A. Jullien, A. Malvache, L. Canova, A. Borot, A. Trisorio, C. G. Durfee, and R. Lopez-Martens, Opt. Lett. 34, 1588 (2009).

8. A. Jullien, O. Albert, G. Chériaux, J. Etchepare, S. Kourtev, N. Minkovski, and S. M. Saltiel, J. Opt. Soc. Am. B 22, 2635 (2005).

9. S. Kourtev, N. Minkovski, L. Canova, A. Jullien, O. Albert, and S. M. Saltiel, J. Opt. Soc. Am. B 26, 1269 (2009).

10. L. Canova, S. Kourtev, N. Minkovski, A. Jullien, R. Lopez-Martens, O. Albert, and S. M. Saltiel, Appl. Phys. Lett. 92, 231102 (2008).

11. G. Petrocelli, E. Pichini, F. Scudieri, and S. Martellucci, J. Opt. Soc. Am. B 10, 918 (1993).

12. A. Jullien, O. Albert, G. Chériaux, J. Etchepare, S. Kourtev, N. Minkovski, and S. M. Saltiel, Opt. Express 14, 2760 (2006).

13. M. Dabbicco, A. M. Fox, G. von Plessen, and J. F. Ryan, Phys. Rev. B 53, 4479 (1996).

14. X. Chen, L. Canova, A. Malvache, A. Jullien, R. LopezMartens, C. Durfee, D. Papadopoulos, and F. Druon, Appl. Phys. B 99, 149 (2010). 